\title{
Attention is captured by distractors that uniquely correspond to controlled objects: An analysis of movement trajectories
}

\author{
James D. Miles • Robert W. Proctor
}

Published online: 3 December 2014

(C) The Psychonomic Society, Inc. 2014

\begin{abstract}
When reaching for a particular target, movements tend to deviate toward distractors. Previously, we have shown that cursor movements deviate to a greater degree toward distractors when the distractor color corresponds to that of the cursor and the target color does not, even when this relationship is task-irrelevant (Miles \& Proctor, 2011). In the present study, we investigated whether this correspondence effect is due to attention capture or to the activation of responses based on the task response rules associated with the colors of the distractor and target (viz. a flanker effect). Participants moved a central rectangular cursor to an upper left or upper right location, depending on the cursor color. The colors of the target (correct response side) and distractor (incorrect response side) were independent from one another and were either corresponding or noncorresponding with respect to the cursor color. In Experiment 1, reaction times were delayed when the distractor color corresponded to that of the cursor, but only when the target color did not correspond to the cursor color. No color correspondence effect was found for movement trajectories or movement times. However, in Experiment 2, when responses were time-pressured, initial movements toward the distractor were much more common when the distractor color exclusively corresponded to the cursor color. On the basis of these results, we argue that attention capture best explains the increased tendency to move a controlled object to a distractor that uniquely shares its features.
\end{abstract}

J. D. Miles $(\bowtie)$

Department of Psychology, California State University, 1250

Bellflower Boulevard, Long Beach, CA 90840-0901, USA

e-mail: jmiles@csulb.edu

R. W. Proctor

Purdue University, West Lafayette, IN, USA
Keywords Attentional capture - Goal-directed movements · Perception and action

When reaching to one of an array of potential targets, movements tend to deviate toward neighboring distractors in other potential target locations (Song \& Nakayama, 2009; Spivey, Grosjean, \& Knoblich, 2005; Tipper, Howard, \& Houghton, 1998; Tipper, Lortie, \& Baylis, 1992; Welsh \& Elliott, 2004). Most of the reaching studies above have placed emphasis on the location, number, and type of distractor. The properties of the object that is controlled during the movement-often some variant of a computer cursor - are largely ignored. However, the correspondence, or match, between the features of the controlled object and those of the intended target and other distractors in the environment may also influence response selection, which would be reflected in response latencies and movement trajectories. These situations are commonplace; for example, most sorting tasks involve identifying some specific feature of an object and, on the basis of that feature, selecting the correct location at which to place the object. The potential goal location may contain features that correspond (match) or do not correspond (mismatch) to those of the object, even if the features are irrelevant to the task itself.

Evidence already exists that certain types of distractors influence movement trajectories when they share physical or conceptual similarities with the targets (e.g., Finkbeiner \& Friedman, 2011; Song \& Nakayama, 2006; Welsh, 2011; Welsh \& Elliott, 2004). For example, Welsh (2011) found that an invalid cue immediately prior to movement to a target location caused movements to deviate in its direction, but only when the cue included the same feature that was used to select the target. Miles and Proctor (2011) also showed that the relation between the cursor and distractor colors influenced responses. Participants moved a colored cursor (red or green) 
to a rectangle on the left or right side of a display (the target) while ignoring a rectangle on the opposite side (the distractor); one of the rectangles was red and the other was green. Responses were slower and deviated toward the distractor side when the distractor matched the color of the cursor, even when the color correspondence was irrelevant to task performance. This color correspondence effect only occurred when the cursor color determined the response ("if the cursor is green, move to the right rectangle; if the cursor is red, move to the left rectangle," or vice versa). The authors concluded that color correspondence effects transpire between a controlled cursor and distractor, but only when participants are forced to first attend to the cursor color.

Two mechanisms could potentially underlie the correspondence effects between cursors and distractors observed in Miles and Proctor's (2011) study. First, attention may be captured by distractors that uniquely share the color of the cursor, which in turn activates a response in their direction. Once attention is captured by the color-corresponding distractor, a response code for a movement toward it is activated, which in turn interferes with execution of the response toward the target (e.g., Becker, 2010; Song \& Nakayama, 2009; Welsh, 2011; Welsh, Elliott, \& Weeks, 1999). In this case, competing responses toward multiple targets increase in activation until one of them reaches some threshold, and the amount of activation of a particular response is at least partially determined by the amount of attention the target of that response receives. Although some debate exists, there is consistent evidence that attention capture is contingent on taskrelevant parameters (Folk, Leber, \& Egeth, 2002; Folk \& Remington, 1998; Folk, Remington, \& Johnston, 1992). Such contingent attention capture may be specific to a particular feature, such as a single color, rather than to the general feature category (e.g., all colors). This view has been substantiated by prior work showing attention capture following the presentation of a cue in the color that indicated a task-relevant target, but no similar capture for other colors (Ansorge \& Heumann, 2003, 2004; Lamy, Leber, \& Egeth, 2004; Leblanc, Prime \& Jolicœur, 2008). Extending the logic of contingent capture to controller correspondence effects, attention capture by a particular target or distractor may be contingent on the features of the controller. In this case, attention is initially focused on the task-relevant color of the controlled object (the cursor), which in turn makes any other stimulus matching that color more salient. The now-salient corresponding stimulus then captures attention and elicits response activation toward its location. We refer to this as the attention-mediated account.

Alternatively, the color of any target or distractor on the display may directly activate the response associated with its color on the basis of the task instructions, without any need to invoke attention capture as a mediating factor. When the color of a particular target or distractor is associated with a response that is different from that of the task-informative cursor color (noncorresponding), then response conflict would occur. For example, if the response rule is "Move a GREEN cursor to the RIGHT target and move a RED cursor to the LEFT target," then any stimulus that is green will also activate a right response to some degree, and any red stimulus will activate a left response, regardless of whether the stimulus is a distractor or target. In other words, targets and distractors act like flankers that may interfere with selecting the correct response to the cursor color (Baylis \& Driver, 1992; Eriksen \& Eriksen, 1974). Accordingly, flanker effects will be most beneficial to performance when the target and distractor both correspond to the color of the cursor, and therefore all colors on the display indicate the same (correct) response. Conversely, flanker effects will be most detrimental when the target and distractor both do not correspond to the cursor color and indicate a competing response according to task rules. For the sake of simplicity, we refer to this as the rulebased account.

The goal of the present study was to determine whether the increased tendency to move a cursor toward a colorcorresponding distractor is due to attention capture by the distractor or to the color of the distractor directly activating a response in its direction on the basis of the response rules of the task. In Experiment 1, participants moved a cursor to an upper left or upper right rectangle on the display using a computer mouse. The movement direction was determined by the color of the cursor; the rectangle on the correct side, as indicated by the cursor color, was the target, and the other rectangle was the distractor. The correspondences between the colors of the cursor and the target and those of the cursor and the distractor were varied independently from one another. This allowed us to examine the respective contributions of the color correspondences between the cursor and target and the cursor and distractor and the interaction between them.

Using the design above, attention-mediated and rule-based accounts predict specific contrasting behaviors for each possible trial type (see Table 1). According to an attentionmediated account, responses should be drawn toward targets that uniquely share the same color as the cursor. As has been previously stated, in this case, the color of the cursor renders a uniquely color-corresponding target or distractor more salient, and therefore more likely to capture attention and activate a response in its direction. When the cursor and target uniquely correspond (e.g., red cursor, red target, and green distractor), the target is made more salient, and therefore more likely to capture attention and activate a response in its direction. Conversely, a uniquely corresponding cursor and distractor (e.g., red cursor, green target, and red distractor) will activate a response toward the distractor side. If the cursor color corresponds to that of both the target and the distractor, then both target and distractor will be equally likely to capture attention, and there will no longer be a tendency to activate a response in either direction. The distractor and 
Table 1 Each possible color configuration of the stimuli in Experiments 1 and 2, and the resulting response biases predicted by the attention-mediated and rule-based accounts

\begin{tabular}{|c|c|c|c|c|}
\hline Cursor Color & Target & Distractor & Attention-Mediated & Rule-Based \\
\hline & Corresponding & Corresponding & & \\
\hline Red & Red & Red & None & Target \\
\hline \multirow[t]{2}{*}{ Green } & Green & Green & None & Target \\
\hline & Noncorresponding & Noncorresponding & & \\
\hline Red & Green & Green & None & Distractor \\
\hline \multirow[t]{2}{*}{ Green } & Red & Red & None & Distractor \\
\hline & Corresponding & Noncorresponding & & \\
\hline Red & Red & Green & Target & Both \\
\hline \multirow[t]{2}{*}{ Green } & Green & Red & Target & Both \\
\hline & Noncorresponding & Corresponding & & \\
\hline Red & Green & Red & Distractor & Both \\
\hline Green & Red & Green & Distractor & Both \\
\hline
\end{tabular}

target should also be equally likely to capture attention when they are both in the color that does not correspond to the cursor.

Alternatively, according to the rule-based account, the color of any potential target on the display will activate its associated task-related response, regardless of the cursor color. In this case, responses should reflect the greatest response bias toward the distractor when both the target and the distractor are presented in the color that leads to the incorrect response, toward the distractor side. For example, if a green cursor indicates moving to the right side and a red cursor indicates moving to the left, when the cursor is green and both the target and distractor are red (and thus do not correspond with the cursor), responses will be slowest and will deviate most toward the distractor side. Conversely, when both the target and distractor correspond to the cursor color, they will activate the same response as the cursor color and will strongly bias the same response toward the target. When the target and distractor are different colors, response biases toward both the target and distractor sides will be created, leading to only a moderate movement deviation toward the distractor.

\section{Experiment 1}

Method

Participants A group of 34 Introductory Psychology students from Purdue University (20 female, 14 male), 18-23 years of age, participated for experimental credits. Thirty-one of the participants reported being right-handed, and three left-handed. None reported being colorblind.

Apparatus and stimuli On each trial, a colored rectangle appeared at the bottom center of the display (the cursor).
Additionally, a larger red or green rectangle appeared in the top left corner of the display, and a similar rectangle appeared at the top right of the display (the target and distractor; see Fig. 1). The cursor rectangle was $1.8 \mathrm{~cm}$ wide and $1.4 \mathrm{~cm}$ high, and its center was at the center of the screen horizontally and $3.6 \mathrm{~cm}$ from the bottom of the screen. The upper left and upper right rectangles were both $3.7 \mathrm{~cm}$ wide and $3.0 \mathrm{~cm}$ high. The upper left rectangle was located $3.4 \mathrm{~cm}$ from the left edge of the screen $3.6 \mathrm{~cm}$ from the top edge. The center of the upper right rectangle was $3.4 \mathrm{~cm}$ from the right edge of the screen and $3.6 \mathrm{~cm}$ from the top edge. The distance between the center

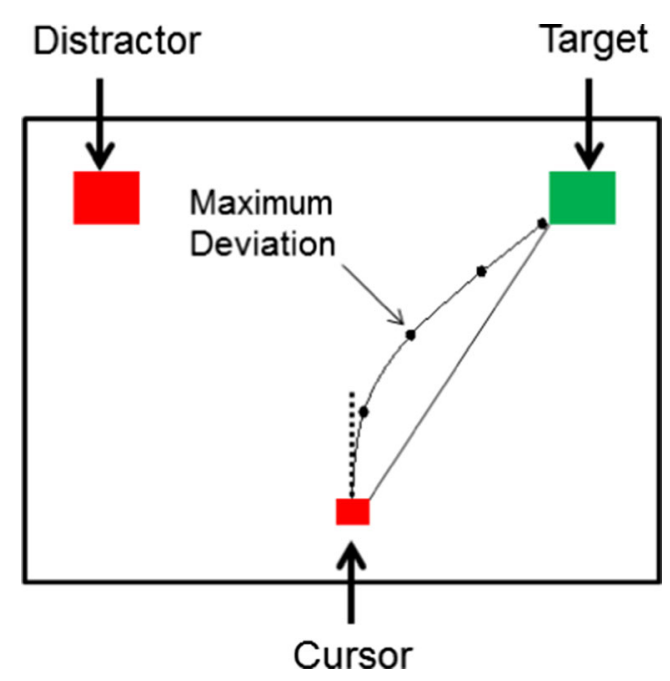

Fig. 1 A sample display and movement trajectory in the experiment. The actual background of the display was black. A computer mouse controlled the movement of the cursor, which was moved to one of two upper boxes, depending on the cursor color. The cursor was either green or red, and in the provided example, the rule is "move a red cursor to the right target, move a green cursor to the left target." In this case, the cursor and target are noncorresponding, and the cursor and distractor are corresponding. Cursor-target and cursor-distractor correspondence were independently varied on a trial-by-trial basis 
of the upper left and upper right rectangles was $31.7 \mathrm{~cm}$, and the diagonal distance between the center of the cursor and the center of each of the upper rectangles was $27.1 \mathrm{~cm}$. The colors of the cursor and the upper left and right rectangles were either red or green and varied independently from one another. The computer mouse used to control the onscreen cursor rectangle at the bottom center of the display was placed on the table in front of the screen and aligned to the horizontal center of the screen. The distance of the center of the mouse from the base of the monitor was approximately $25 \mathrm{~cm}$.

Procedure At the beginning of the experiment, participants were first asked if they had been diagnosed with colorblindness, and they were given a single trial of the Ishihara color vision test (Ishihara, 1993). Following the color vision test, participants were instructed that they would move a cursor using the mouse into the upper left or upper right rectangle, depending on the color of the cursor. For half of the trial blocks, participants used a green cursor-left target, red cursor-right target mapping; the reversed mapping was used for the other trial blocks. Each trial began with the presentation of gray boxes in the cursor and left and right potential target locations, which remained on the screen for $500 \mathrm{~ms}$. These boxes were then replaced by the colored cursor and colored rectangles in the upper left and upper right of the screen. For a random half of the trials in a block, the cursor was red, and for the other half it was green. On equal numbers of trials, the left and right upper rectangles were red and green, and the colors of the left and right rectangles were also corresponding and noncorresponding with respect to the cursor color on equal numbers of trials. We imposed no time limit for completing the response. If the cursor was moved inside the distractor, the boxes disappeared and the words "Incorrect Target!" appeared on the screen for 2,000 ms. After completion of a trial, the gray boxes appeared again, and the next trial began. Participants received eight practice trials, followed by two blocks of 64 trials each of the task, and then two more blocks using the reverse cursor color-target location mapping. Each block contained equal numbers of trials in which the rectangles were both red, both green, left red and right green, and left green and right red.

Analyses Several measures were created from the resulting response data. First, reaction times (RT) were calculated as the amount of time that passed from the onset of the colored cursor and upper rectangles until the following two conditions were met: the cursor had moved more than $1 \mathrm{~cm}$ upward toward the targets from its starting location and the cursor movement had reached a minimum velocity of at least $5 \mathrm{~cm} / \mathrm{s}$. Velocity was calculated as the overall distance that the cursor had moved, in centimeters, over the previous 15 to $20 \mathrm{~ms}$. The movement time (MT) then began immediately when the RT ended and continued until when either the edge of the cursor touched the edge of one of the upper rectangles or the cursor edge was within $2 \mathrm{~cm}$ of the edge of one of the upper rectangles and the velocity had decreased below $5 \mathrm{~cm} / \mathrm{s}$. This decrease in velocity indicated that the initial movement was complete.

Movement trajectory An example cursor trajectory is shown in Fig. 1. Mouse movements that controlled the cursor were sampled approximately every 1 to $2 \mathrm{~ms}$, and they recorded the location of the cursor in relation to its starting position, in millimeters. All cursor locations were recorded throughout the movement duration. The time since the onset of the movement and the location of the cursor was then determined at each $25 \%$ of the MT. A direct path for each movement was also calculated as a straight line between the starting location of the cursor and the location of the cursor when it first touched the target.

On the basis of these data, two measurements of movement trajectory were taken. The percentage of initial movement errors (IMerror) was calculated as the number of trials on which the location of the cursor was on the distractor side of the screen after $25 \%$ of the movement had been completed (i.e., on the distractor side of the dotted midline in Fig. 1), indicating that the initial movement of the cursor was toward the distractor. Second, the maximum deviation was the deviation of the quartile time bin with the greatest distance from the direct path. Deviations toward the distractor side were recorded as positive values, and deviations farther toward the target side were recorded as negative values. The maximum deviation was averaged across all trials for each participant.

\section{Results}

Trials on which the total response time $(\mathrm{RT}+\mathrm{MT})$ was greater than $1,500 \mathrm{~ms}$ were removed from the analysis (for a similar cutoff criterion, see Song \& Nakayama, 2009). This cutoff was chosen in order to focus on trials in which responses were initiated and executed promptly. One participant was found to have a large percentage of trials in which responses were not completed in time (over $50 \%$ of trials), and this participant was removed from further analysis. For the 33 remaining participants, the response-time cutoff criterion led to the removal of $10 \%$ of all trials. Trials in which the cursor was moved completely into the distractor, which constituted $1 \%$ of all trials, were also removed.

Reaction time RTs were analyzed for the factors of Cursor-Target Color Correspondence (corresponding vs. noncorresponding), Cursor-Distractor Color Correspondence (corresponding vs. noncorresponding), and Target Side (left vs. right). RTs were shorter when the cursor and target colors corresponded $(M=507 \mathrm{~ms})$ than when they did not 
$(M=522 \mathrm{~ms}), F(1,32)=6.43, p<.05, \eta_{\mathrm{p}}^{2}=.17$. Although RTs also tended to be longer when the cursor and distractor colors corresponded $(M=517 \mathrm{~ms})$ than when they did not $(M=511 \mathrm{~ms})$, this difference was not statistically significant, $F(1,32)=2.03, p=.16, \eta_{\mathrm{p}}{ }^{2}=.06$. However, as can be seen in Fig. 2a, the interaction between cursor-target and cursordistractor correspondence was significant, $F(1,32)=4.96$, $p<.05, \eta_{\mathrm{p}}{ }^{2}=.13$. A paired-sample $t$ test indicated that when the cursor and target were noncorresponding colors, RTs were significantly longer, by $16 \mathrm{~ms}$, when the cursor corresponded to the distractor color than when it did not, $t(32)=2.49$, $p<.05$. Furthermore, RTs were longer when the distractor was corresponding and the target was noncorresponding $(M=530 \mathrm{~ms})$ than when they both corresponded to the cursor $(M=505 \mathrm{~ms}), t(32)=3.06, p<.05$, indicating that RTs
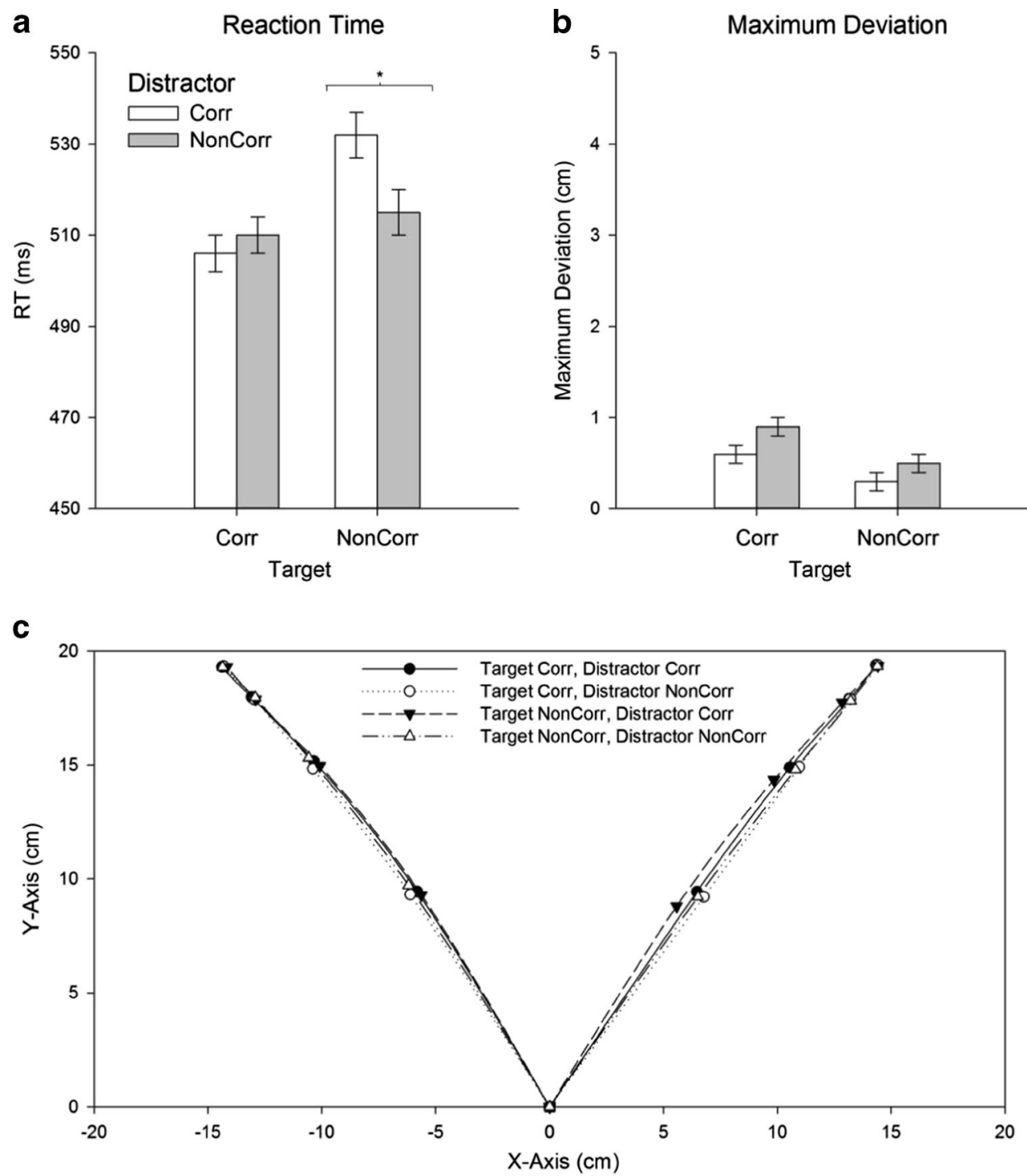

Fig. 2 (a) Reaction times and (b) maximum deviations for each combination of cursor-target and cursor-distractor correspondence in Experiment 1. Error bars represent within-subjects standard errors, and asterisks indicate significant differences $(p<.05)$. (c) Experiment 1 noncorresponding

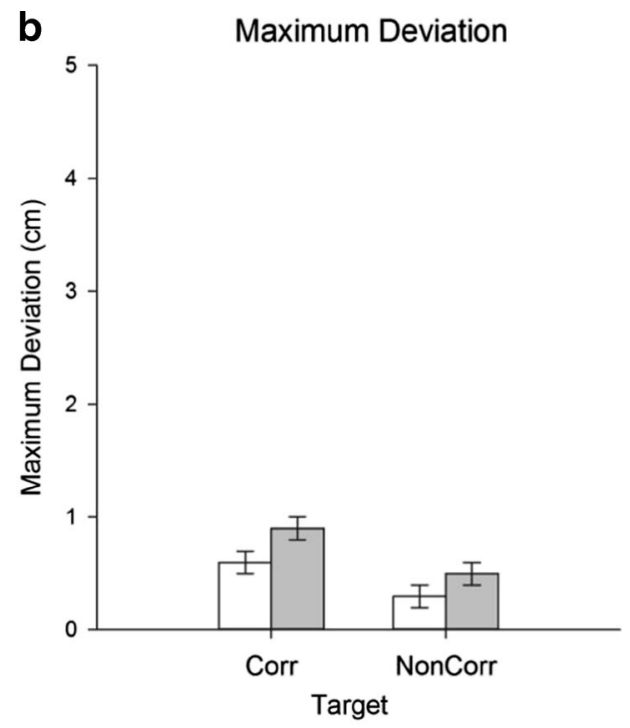

were specifically delayed when the distractor uniquely corresponded to the cursor. When the cursor and distractor were noncorresponding, we found no benefit of a corresponding relative to a noncorresponding target; there was also no RT benefit when only the target corresponded, relative to when both the target and distractor corresponded $(t \mathrm{~s}<1, p \mathrm{~s}>.45)$. Target side had no significant main effect on RTs, nor did it interact with any other factors (all $\left.F_{\mathrm{s}}<1.5, p \mathrm{~s}>.24\right)$.

Maximum deviations The maximum movement deviation away from the target is shown in Fig. 2b, and the movement trajectories are shown in Fig. 2c. Although maximum deviations of the movement trajectory toward the distractor (MaxDev) were smaller when the colors of the cursor and

movement trajectories toward left or right targets, averaged at every $25 \%$ of the movement time for each combination of cursor-target and cursor-distractor correspondence. Corr $=$ corresponding, NonCorr $=$ 
target were corresponding $(M=0.5 \mathrm{~cm})$ than when they were noncorresponding $(M=0.7 \mathrm{~cm})$, this difference was not significant, $F(1,32)=3.59, p=.07, \eta_{\mathrm{p}}{ }^{2}=.10$. A main effect was found for cursor-distractor correspondence, such that the maximum deviation toward the distractor was greater when the cursor and distractor corresponded $(M=0.8 \mathrm{~cm})$ than when they $\operatorname{did} \operatorname{not}(M=0.4 \mathrm{~cm}), F(1,32)=12.48, p<.05$, $\eta_{\mathrm{p}}{ }^{2}=.28$. No interaction between cursor-target and cursordistractor correspondence emerged, $F<1$. Maximum deviations were larger for left targets $(M=0.9 \mathrm{~cm})$ than for right targets $(M=0.3 \mathrm{~cm}), F(1,32)=8.81, p<.05, \eta_{\mathrm{p}}{ }^{2}=.22$, but the effect of target side did not interact with cursor-target correspondence, $F(1,32)=2.39, p=.13, \eta_{\mathrm{p}}^{2}=.07$, or cursordistractor correspondence, $F(1,32)=1.29, p=.27, \eta_{\mathrm{p}}{ }^{2}=.04$, and there was no three-way interaction between the factors, $F(1,32)=1.53, p=.23, \eta_{\mathrm{p}}{ }^{2}=.05$.

Initial movement errors As is shown in Table 2, the percentage of initial movements erroneously made toward the distractor side (IMerror) was slightly lower when the cursor and target were corresponding colors $(M=3 \%)$ than when they were noncorresponding $(M=5 \%), F(1,32)=6.35$, $p<.05, \eta_{\mathrm{p}}{ }^{2}=.17$. Conversely, IMerror was greater when the cursor and distractor colors corresponded $(M=5 \%)$ than when they $\operatorname{did} \operatorname{not}(M=4 \%), F(1,32)=5.47, p<.05$, $\eta_{\mathrm{p}}{ }^{2}=.15$. We observed no significant Cursor-Target $\times$ Cursor-Distractor Correspondence interaction, $F(1,32)=$ $2.31, p=.14, \eta_{\mathrm{p}}{ }^{2}=.07$, and no other main effects or interactions were significant, $F \mathrm{~s}<1.8, p \mathrm{~s}>.26$.

Movement time Although MTs were slightly shorter when the cursor and target colors corresponded $(M=421 \mathrm{~ms})$ than when they did not $(M=430 \mathrm{~ms})$, this difference was not significant, $F(1,32)=2.77, p=.11, \eta_{\mathrm{p}}{ }^{2}=.08$. All other main effects and interactions also did not reach significance (all $F \mathrm{~s}<1.7, p \mathrm{~s}>.2$ ).

Table 2 Mean and within-subjects standard errors for movement time (MT) in milliseconds and for percentages of initial movements toward the distractor (IMerror) for the factors of cursor-target and cursor-distractor correspondence in Experiments 1 and 2

\begin{tabular}{|c|c|c|c|c|}
\hline \multirow[b]{3}{*}{ Distractor } & \multicolumn{4}{|l|}{ Target } \\
\hline & \multicolumn{2}{|l|}{ MT } & \multicolumn{2}{|l|}{ IMerror } \\
\hline & Corr & NonCorr & Corr & NonCorr \\
\hline \multicolumn{5}{|c|}{ Experiment 1} \\
\hline Corr & $424(5)$ & $425(6)$ & $3 \%(0.5 \%)$ & $6 \%(0.7 \%)$ \\
\hline NonCorr & $414(5)$ & $431(5)$ & $3 \%(0.6 \%)$ & $4 \%(0.5 \%)$ \\
\hline \multicolumn{5}{|c|}{ Experiment 2} \\
\hline Corr & $373(7)$ & $418(7)$ & $18 \%(0.9 \%)$ & $28 \%(1.4 \%)$ \\
\hline NonCorr & $373(6)$ & $381(6)$ & $17 \%(1.0 \%)$ & $21 \%(1.0 \%)$ \\
\hline
\end{tabular}

Correlations Since performance costs were primarily observed when the cursor and distractor colors corresponded but the cursor and target colors did not, these trials were further analyzed to determine whether the performance measures of RT, MT, IMerror, and MaxDev were related to one another. Several significant correlations were notable. First, we found a negative correlation between RT and MT, $r(33)=-.38, p<.05$, signifying a performance tradeoff between these two response stages. Second, the correlation between IMerror and MaxDev was very large, $r(33)=.79$, $p<.05$, indicating that the average maximum deviation was larger when there were a greater number of initial movements toward the distractor side. Thus, the influence of a uniquely corresponding distractor is likely only on the initial vector of the movement, which then influences the subsequent maximum deviation of the movement from the direct target path.

\section{Discussion}

As is predicted by the attention-mediated account, RTs were longest when the distractor color corresponded with that of the cursor but the target color did not. Furthermore, as is shown in Fig. 2a, RTs were not different when the target and distractor both corresponded to the cursor than when they both did not. These results imply that when the distractor uniquely shared the same color as the cursor, it captured attention in its direction, which in turn activated a response in the direction of the distractor, delaying initiation of the response toward the target. According to the rule-based, flanker-type response activation account, responses should have been slowest when both the target and distractor were noncorresponding with the distractor, leading both to directly activate the incorrect response, toward the distractor, on the basis of their color - this was not the case.

The attention-mediated account also predicts a benefit to performance in the direction of the target when the target uniquely corresponds to the cursor, but, as is shown in Fig. 2a, this was not the case. One possible reason for this result is that the benefits of a uniquely corresponding target are simply much smaller than the costs of a uniquely corresponding distractor. Specifically, attention capture by a uniquely corresponding distractor requires attention to be reoriented to the target prior to the initiation of a movement to the target. Conversely, a uniquely corresponding target provides little additional benefit when attention is already oriented toward the target through endogenous control.

\section{Experiment 2}

Although the results of Experiment 1 were consistent with an attention-mediated account, the effect occurred just for RTs, 
which provides only indirect evidence for the activation of a response toward the distractor when it uniquely corresponds to cursor color. The relatively long mean RT coupled with the negative correlation between RT and MT suggests that participants were delaying movement initiation until the response conflict was resolved. If so, it should be possible to shift the influence of the distractor color on performance to the movement itself if participants are required to initiate the movement sooner. Therefore, in Experiment 2, a strict time limit was placed on movements in order to encourage the onset of movement to take place before any response conflict could be resolved. In this case, it was expected that any influence of color correspondence between the cursor and the target or distractor would be evident in the MTs and trajectories rather than in the RTs.

In addition to the new time constraint, a baseline condition was included during the first two blocks of the experiment, in which participants moved a white rectangular cursor to a white rectangular target on the upper left or upper right of the display. No distractor was presented in this condition. The rationale for including this baseline was to determine whether the mere existence of a distractor influenced response performance in the present experiment - initial movements as well as maximum movement deviations toward the distractor side might simply represent the natural curvature of the movement toward the target. If this were indeed the case, then a similar curvature in the movement should be found in the baseline condition, in which a target but no distractor appeared on the display during a trial.

\section{Method}

Participants A group of 31 Introductory Psychology students from California State University, Long Beach (18 female, 13 male), 18-21 years of age, participated for experimental credit. Thirty of the participants were right-handed, and one was left-handed. None reported being colorblind.

Apparatus and stimuli The configuration of the cursor, target, and distractor were the same as in Experiment 1. However, the experiment was run on slightly different computer hardware. Of note, the monitors were widescreen and 22 in. in diameter, rather than $17 \mathrm{in}$. and standard in dimensions. This change slightly altered the metric sizes and locations of the cursor, target, and distractor.

The cursor rectangle was $2.0 \mathrm{~cm}$ wide and $1.5 \mathrm{~cm}$ high, and its center was at the center of the screen horizontally and $6.0 \mathrm{~cm}$ from the bottom of the screen. The upper left and upper right rectangles were both $4.5 \mathrm{~cm}$ wide and $3.0 \mathrm{~cm}$ high. The upper left rectangle was located $4 \mathrm{~cm}$ from the left edge of the screen and $4 \mathrm{~cm}$ from the top edge. The center of the upper right rectangle was $4.0 \mathrm{~cm}$ from the right edge of the screen and $4.0 \mathrm{~cm}$ from the top edge. The distance between the center of the upper left and upper right rectangles was $41 \mathrm{~cm}$, and the diagonal distance between the center of the cursor and the center of each of the upper rectangles was $28.0 \mathrm{~cm}$. All other aspects of the design were identical to those in Experiment 1.

\section{Procedure}

The procedure was identical to that of Experiment 1, with the following exceptions. First, a time constraint was imposed on all trials. If the RT on a trial was greater than $500 \mathrm{~ms}$ or the RT and MT together took longer than $2,000 \mathrm{~ms}$, the trial was immediately ended and the message "Too Slow! Please Respond Faster!" appeared at the center of the screen. Any trials that were not completed in time were discarded and rerun at the end of the trial block. Since the time constraint made the trials more mentally demanding, each trial was followed by a screen with the text "Click the mouse button when ready for the next trial," which remained on the screen until the mouse button was pressed.

Second, a new baseline condition was added in which the cursor was a white rectangle and a single white rectangle target appeared at either the upper left or upper right of the display. No distractor rectangle appeared in the opposing upper location. Participants were instructed to simply move the cursor from its starting position into the target as quickly as they could. Participants performed two blocks of 48 trials each of the baseline condition at the beginning of the experiment. The target was presented equal numbers of times in the left and right upper positions during both baseline blocks. Responses in the baseline condition were analyzed to determine: (1) whether movement trajectories would show a general deviation away from the target, regardless of whether a distractor was included, and (2) whether movement deviations in conditions with a distractor would be significantly greater than deviations without a distractor. Since the baseline condition was included in order to compare movement deviations when moving to a target with and without a distractor, only the resulting maximum deviations were compared to the data in the other conditions.

\section{Results}

Trials in which the edge of the cursor touched the edge of the distractor, indicating a response on the incorrect side, were removed (3.3\% of all trials). A total of $7.3 \%$ of all trials had RTs greater than $500 \mathrm{~ms}$ or a total combined RT and MT of 2,000 ms; they were immediately stopped and also removed from the analysis.

Reaction time As expected, with a time constraint imposed, no significant effect of cursor-target or cursor-distractor color correspondence was found for RTs $\left(F_{\mathrm{S}}<1, p \mathrm{~s}>.66\right)$, nor did their interaction reach significance, $F(1,30)=2.87, p=.10$, $\eta_{\mathrm{p}}{ }^{2}=.09$ (Fig. 3a). The RTs for left targets were slightly faster 

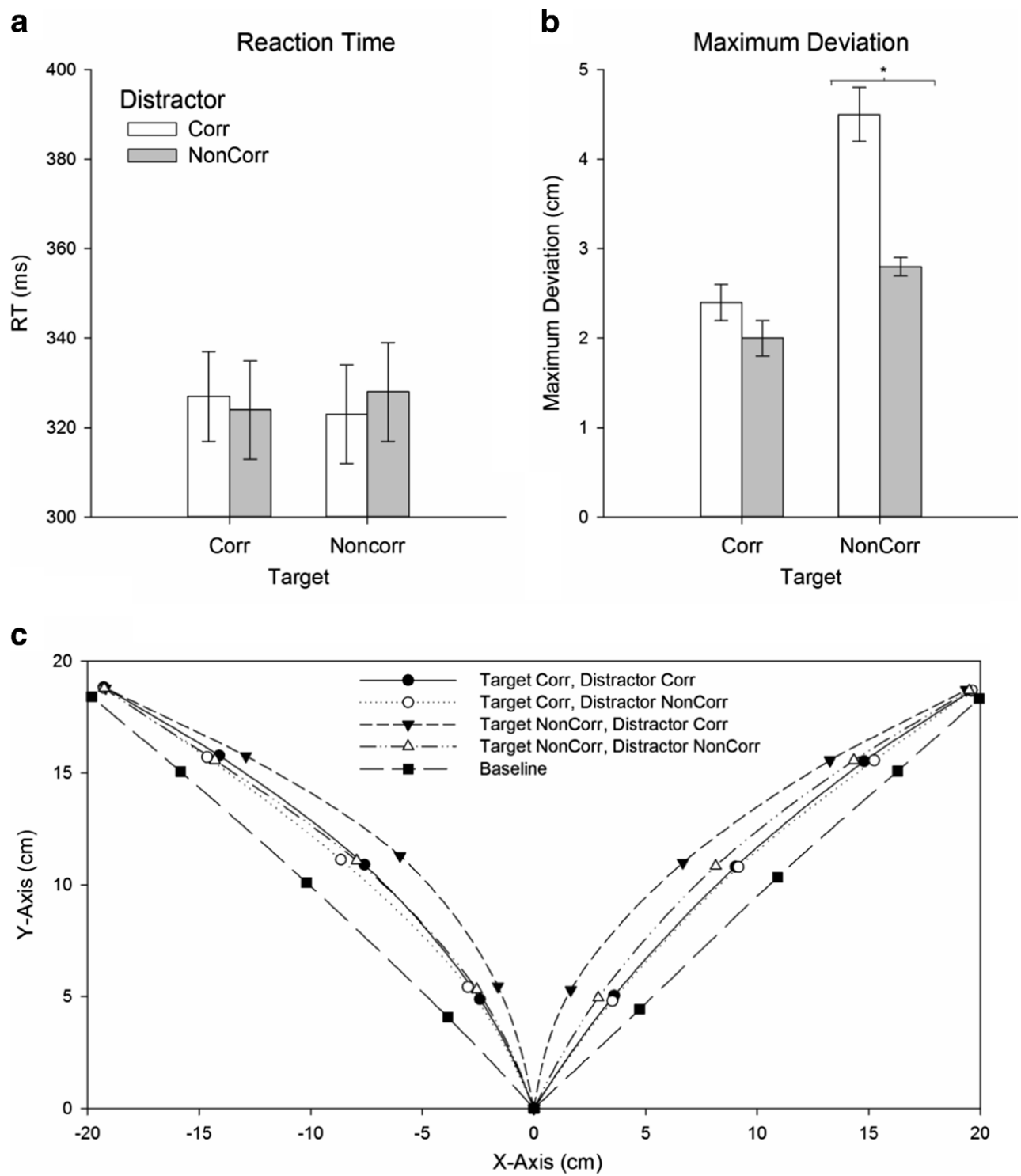

Fig. 3 (a) Reaction times and (b) maximum deviations for each combination of cursor-target and cursor-distractor correspondence in Experiment 2. Error bars represent within-subjects standard errors, and asterisks indicate significant differences $(p<.05)$. (c) Experiment 2 movement trajectories toward left or right targets, averaged at every $25 \%$ of the movement time for each combination of cursor-target and cursor-distractor correspondence, as well as for the baseline condition of moving a white cursor to a white target with no distractor than those to right targets, but this difference was not significant, $F(1,30)=2.31, p=.14, \eta_{\mathrm{p}}{ }^{2}=.07$. Target side did not interact with cursor-target correspondence, $F(1,30)=3.92, p$ $=.06, \eta_{\mathrm{p}}{ }^{2}=.12$, cursor-distractor correspondence, $\quad F<1$, or their combination, $F<1$.

Maximum deviations Maximum deviations (MaxDev) and movement trajectories are shown in Fig. 3b and c, respectively. MaxDev toward the distractor side was smaller when the cursor and target colors corresponded $(M=2.2 \mathrm{~cm})$ than when they $\operatorname{did} \operatorname{not}(M=3.6 \mathrm{~cm}), F(1,30)=38.09, p<.05, \eta_{\mathrm{p}}{ }^{2}=.56$, and the MaxDev was larger when the cursor and distractor colors corresponded $(M=3.4 \mathrm{~cm})$ than when they did not $(M=2.4 \mathrm{~cm}), F(1,30)=16.78, p<.05, \eta_{\mathrm{p}}{ }^{2}=.36$. These correspondence effects were mediated by a significant cursortarget and cursor-distractor correspondence interaction (Fig. 3b), $F(1,30)=10.80, p<.05, \eta_{\mathrm{p}}{ }^{2}=.27$.

In concordance with the RT results in Experiment 1, when the target was noncorresponding, MaxDev was greater when the distractor corresponded with the cursor $(M=4.4 \mathrm{~cm})$ than when it did not $(M=2.8 \mathrm{~cm}), t(30)=4.53, p<.05$, and MaxDev was greater with a corresponding distractor and noncorresponding target $(M=4.4 . \mathrm{cm})$ than with a corresponding distractor and corresponding target $(M=2.4 \mathrm{~cm}), t(30)=5.51, p<.05$, indicating that this movement bias was greatest when the distractor was uniquely corresponding to the cursor.

When the cursor and distractor were noncorresponding, MaxDev was lower when the cursor and target were 
corresponding $(M=2.0 \mathrm{~cm})$ than when they were noncorresponding $(M=2.8 \mathrm{~cm}), t(30)=3.96, p<.05$, reflecting a bias to move toward the target side. Although MaxDev with a corresponding target and a noncorresponding distractor $(M=2.0 \mathrm{~cm})$ was lower than with a corresponding target and a corresponding distractor $(M=2.8 \mathrm{~cm})$, this difference was not significant, $t(30)=1.55, p=.13$, suggesting a bias to move toward a corresponding target, but it did not matter whether the target was uniquely corresponding or not.

Finally, the MaxDev was overall greater for left $(3.3 \mathrm{~cm})$ than for right $(2.5 \mathrm{~cm})$ targets, $F(1,30)=8.57, p<.05$, $\eta_{\mathrm{p}}{ }^{2}=.22$. Target side also interacted with cursor-target correspondence, $F(1,30)=5.50, p<.05, \eta_{\mathrm{p}}{ }^{2}=.16$, but not with cursor-distractor correspondence, and we found no three-way interaction between the factors (both $F_{\mathrm{S}}<1, p \mathrm{~s}>.43$ ).

Initial movement direction The pattern of IMerrors closely resembled the maximum deviations (Table 2). Participants made fewer initial movements toward the distractor when the cursor and target colors corresponded $(M=17 \%)$ than when they did not $(M=24 \%), F(1,30)=34.52, p<.05$, $\eta_{\mathrm{p}}{ }^{2}=.54$, and more initial movements toward the distractor when the cursor and distractor colors corresponded $(M=23 \%)$ than when they did not $(M=19 \%), F(1,30)=$ $8.36, p<.05, \eta_{\mathrm{p}}{ }^{2}=.22$. Cursor-target and cursor-distractor correspondence once again interacted, $F(1,30)=5.74$, $p<.05, \eta_{\mathrm{p}}{ }^{2}=.16$, and paired-sample comparisons showed a pattern similar to that for MaxDev. With noncorresponding targets, we observed more initial movements toward the distractor when it corresponded with the cursor color than when it did not $(M \mathrm{~s}=28 \%$ and $21 \%$, respectively), $t(30)=$ $3.35, p<.05$, and IMerrors were greater when the distractor uniquely corresponded to the cursor $(M=28 \%)$ than when both the distractor and target corresponded to the cursor $(M=18 \%), t(30)=5.01, p<.05$. When the distractor was noncorresponding, IMerrors were lower when the target and cursor corresponded $(M=17 \%)$ than when they did not $(M=21 \%), t(30)=2.46, p<.05$, but the benefit of a corresponding target was the same, regardless of whether the distractor was corresponding with the cursor $(M=18 \%)$ or $\operatorname{not}(M=17 \%), t(30)=0.49, p=.63$. No other main effects or interactions were significant $(F \mathrm{~S}<1.2, p \mathrm{~s}>.29)$.

Movement time As with the IMerrors and maximum deviations, MT was significantly shorter when the cursor and target colors corresponded $(M=373 \mathrm{~ms})$ than when they did not $(M=400 \mathrm{~ms}), F(1,30)=11.20, p<.05, \eta_{\mathrm{p}}{ }^{2}=.27$, and MTs were longer when the cursor and distractor corresponded $(M=396 \mathrm{~ms})$ than when they $\operatorname{did} \operatorname{not}(M=377 \mathrm{~ms}), F(1$, $30)=6.35, p<.05, \eta_{\mathrm{p}}{ }^{2}=.18$ (Table 2). These main effects were likely driven by the significant interaction between cursor-target and cursor-distractor correspondence, $F(1,30)=$ 10.28, $p<.05, \eta_{\mathrm{p}}{ }^{2}=.26$. Paired-sample $t$ tests showed that when the cursor and target colors were noncorresponding, MTs were longer when the cursor and distractor colors corresponded $(M=418 \mathrm{~ms})$ than when they did not $(M=381 \mathrm{~ms}), t(30)=3.95, p<.05$, and the cost of a corresponding distractor was greater when it was uniquely corresponding $(M=418 \mathrm{~ms})$ than when the target also corresponded to the cursor $(M=373 \mathrm{~ms}), t(30)=4.10$, $p<.05$. When the distractor and cursor were noncorresponding, no MT benefit was found when the cursor and target were corresponding $(M=373 \mathrm{~ms})$ relative to when they were not $(M=381 \mathrm{~ms})$, and it did not matter whether or not the target and cursor were uniquely corresponding (both $M \mathrm{~s}=373 \mathrm{~ms}), t \mathrm{~s}<1, p \mathrm{~s}>.35)$.

Baseline comparison of maximum deviations One-sample $t$ tests of the maximum deviations for left and right targets in the baseline conditions determined that trajectories without a distractor were not significantly different from a straight line for movements to the right target $(M=-0.1 \mathrm{~cm}), t(30)=0.63$, $p=.53$; however, movements to the left target slightly deviated away from a straight line and toward the right side of the display $(M=0.5 \mathrm{~cm}), t(30)=2.58, p<.05$. This greater deviation for left than for right target responses was also found in trials with distractors in both Experiments 1 and 2, indicating that this effect is likely a kinematic effect of moving the mouse to the right and to the left with the right hand controlling the mouse. Paired-sample $t$ tests comparing the maximum deviation of movements to left targets in the baseline condition to all four left-target conditions from the earlier analysis found that all conditions with distractors had greater maximum deviations toward the distractor side than did the baseline condition (all $t \mathrm{~s}>4.48$, all $p \mathrm{~s}<.001$ ). Right-target conditions also showed greater maximum deviations in the conditions with a distractor versus the baseline condition (all $t \mathrm{~s}>3.91, p \mathrm{~s}<.001$ ).

Correlations As in Experiment 1, performance costs primarily occurred when the cursor and target colors were noncorresponding and the cursor and distractor colors were corresponding. Therefore, a Pearson correlation was again run to examine the relation between the performance measures in this condition. A negative correlation occurred between RT and MT, $r(31)=-.81, p<.05$, which likely reflects ongoing response selection. When RTs are short, any unresolved response selection is completed during the movement, slowing down MTs. A large positive correlation was found between IMerrors and MaxDev, $r(31)=.89, p<.05$. This again indicated that the maximum deviation of the movement was largely determined by the initial movement trajectory. Initial movement errors were also correlated with MTs, $r(31)=.55$, $p<.05$, likely reflecting the increased time needed to reorient the trajectory toward the correct target following an initial movement toward the distractor side. 


\section{Discussion}

As is shown by the longer MTs and increased IMerrors and maximum deviations, the time constraint in Experiment 2 shifted much of the effect of cursor-target and cursordistractor correspondence effects into the movement itself, likely because response selection was not complete prior to movement onset. The pattern of results remains consistent with the attention-mediated account: Participants were more likely to initiate movement toward a color-corresponding distractor when the target was noncorresponding, indicating a strong influence of a uniquely corresponding distractor on early response selection. In fact, when the cursor color corresponded to the distractor but not the target, the number of erroneous initial movements increased by nearly $10 \%$ relative to the other display arrangements. Again, when both the target and distractor were noncorresponding, and therefore both indicated a response toward the distractor side, the IMerror and maximum deviations were small, indicating that it is unlikely that the target and distractor colors directly activated responses toward the distractor. A similar correspondence effect was found between the cursor and target: Movements were most direct toward a corresponding target. However, the tendency to make more direct movements toward the target did not require the target to uniquely correspond with the cursor. As was described in the Discussion for Experiment 1, the benefit of a unique correspondence effect between the cursor and target may have been much smaller or nonexistent because attention was already oriented toward the target in a controlled, endogenous manner.

\section{General discussion}

The goal of the present study was to examine whether correspondence effects between the color of a controlled cursor and target and the cursor and distractor are due to attentionmediated or direct rule-based activation of responses, similar to those found in flanker tasks. If the observed correspondence effects are due to attention capture, then movements should deviate toward the distractor to the greatest degree when the distractor is made most salient - that is, when the distractor color uniquely matches that of the cursor. If the correspondence effects are due to rule-based response activation, then deviation toward the distractor should be greatest when both the target and distractor colors do not correspond to the cursor, and therefore are associated with responses toward the distractor side. The results are strongly consistent with the former interpretation - following the capture of attention by the distractor, a response in its direction becomes active and must be resolved, leading to delays in response initiation (Exp.
1) or, under time pressure (Exp. 2), initial movement deviations toward the distractor.

Our results are in line with a "horse race" response activation account (Welsh \& Elliott, 2004; Welsh et al., 1999). At the onset of the task, two competing responses became active-one toward the target location and one toward the distractor. These responses raced to reach an activation threshold that would determine the initial direction of the response. Although the correct target response often won this race, the activation of the distractor response either delayed the response or, occasionally, won out. When the distractor uniquely shared the color of the cursor, it became more salient, increasing its chance of capturing attention, which increased activation of the response in its direction. This led the distractor response to more often win the race, causing more erroneous initial movements toward the distractor that were subsequently corrected following the onset of movement (Folk et al., 1992; Geng \& DiQuattro, 2010). Consistent with this account, the benefit of attention capture toward a uniquely corresponding target was negligible, because the rules of the task itself already oriented attention toward the target location.

It is also noteworthy that, when compared to the baseline condition in Experiment 2, the trajectories of all movements tended to deviate toward the distractor side to some extent. As is shown in Fig. 3c, this deviation occurred even when the distractor color was noncorresponding to the cursor, and therefore its color indicated a response in the direction of the target. By itself, this result is not surprising-previous research has consistently shown that response competition occurs whenever multiple potential targets are present (Song \& Nakayama, 2006; Tipper et al., 1998; Tipper et al., 1992; Welsh \& Elliott, 2004). This result is also strongly in line with contingent-attentional-capture accounts, since the distractor and target were always colors that were relevant for response selection when presented in the cursor. However, contingent capture alone cannot explain the greater deviation toward a distractor that was the same color as the cursor. Rather, the unique influence of a color-corresponding distractor was likely due to additional attention capture caused by color priming. This color-priming effect is similar to what was found by Finkbeiner, Song, Nakayama, and Caramazza (2008); when participants were first presented with a masked prime of the word "red" or "green," their movements on a subsequent discrimination task deviated toward a target that matched the color represented in the prime word. Importantly, the masked prime itself did not directly activate a specific response, but rather biased a response toward a subsequent matching target location. Again, it is likely that, both with the masked primes in Finkbeiner et al. and the cursor in our experiments, colorcorresponding stimuli captured attention, which in turn led to response activation in their direction.

Finally, attention may influence movement trajectories at two response stages (see Godijn \& Theeuwes, 2002; 
Theeuwes \& Godijn, 2002). First, attention may be captured by a distractor that uniquely corresponds to the cursor, leading to early response competition. In this case, at the onset of a trial, attention is immediately directed toward the distractor and quickly activated a competing response in the distractor's direction. Second, the corresponding distractor color may make it more difficult to disengage attention after the onset of a response. The results of both experiments indicate that cursor correspondence effects are due to attention capture that occurs prior to the onset of the movement rather than to a problem of disengaging attention. In Experiment 1, the effect of cursor-distractor color correspondence is nearly entirely in the RTs, and even in Experiment 2, with a strict emphasis on the early onset of movement, the influence of the distractor is on the initial movement direction rather than on later components. Furthermore, as was shown in the correlations between performance measures, the maximum deviation of the movement toward a uniquely corresponding distractor was largely related to the initial movement direction, indicating that the influence of the distractor was largely at the onset of the movement.

In summary, the present results show that the color of a controlled object made a distractor that uniquely shared that color more salient, causing that distractor to capture attention, which in turn increased the chance of initiating a response in the distractor's direction. Although the influence of attention on response activation is well established, we believe that the potential for "feature attraction" between controlled objects and distractors caused by attention capture is a largely unexplored phenomenon that may have a wide range of effects on response behavior.

Author Note Preparation of this article was supported in part by NASA Cooperative Agreement NNX09AU66A, Group 5 University Research Center: Center for the Human Factors in Advanced Aeronautics Technologies (Brenda Collins Technical Monitor).

\section{References}

Ansorge, U., \& Heumann, M. (2003). Top-down contingencies in peripheral cuing: The roles of color and location. Journal of Experimental Psychology: Human Perception and Performance, 29, 937-948. doi:10.1037/0096-1523.29.5.937

Ansorge, U., \& Heumann, M. (2004). Peripheral cuing by abrupt-onset cues: The influence of color in S-R corresponding conditions. Acta Psychologica, 116, 115-143. doi:10.1016/j.actpsy.2004.01.001

Baylis, G. C., \& Driver, J. (1992). Visual parsing and response competition: The effect of grouping factors. Perception \& Psychophysics, 51, 145-162. doi:10.3758/BF03212239

Becker, S. I. (2010). The role of target-distractor relationships in guiding attention and the eyes in visual search. Journal of Experimental Psychology: General, 139, 247-265. doi:10.1037/a0018808

Eriksen, B. A., \& Eriksen, C. W. (1974). Effects of noise letters upon the identification of a target letter in a nonsearch task. Perception \& Psychophysics, 16, 143-149. doi:10.3758/BF03203267
Finkbeiner, M., \& Friedman, J. (2011). The flexibility of nonconsciously deployed cognitive processes: Evidence from masked congruence priming. PLoS ONE, 6, e17095. doi:10.1371/journal.pone.0017095

Finkbeiner, M., Song, J. H., Nakayama, K., \& Caramazza, A. (2008). Engaging the motor system with masked orthographic primes: A kinematic analysis. Visual Cognition, 16, 11-22.

Folk, C. L., Leber, A. B., \& Egeth, H. E. (2002). Made you blink! Contingent attentional capture produces a spatial blink. Perception \& Psychophysics, 64, 741-753. doi:10.3758/BF03194741

Folk, C. L., \& Remington, R. (1998). Selectivity in distraction by irrelevant featural singletons: Evidence for two forms of attentional capture. Journal of Experimental Psychology: Human Perception and Performance, 24, 847-858. doi:10.1037/0096-1523.24.3.847

Folk, C. L., Remington, R. W., \& Johnston, J. C. (1992). Involuntary covert orienting is contingent on attentional control settings. Journal of Experimental Psychology: Human Perception and Performance, 18, 1030-1044. doi:10.1037/0096-1523.18.4.1030

Geng, J. J., \& DiQuattro, N. E. (2010). Attentional capture by a perceptually salient non-target facilitates target processing through inhibition and rapid rejection. Journal of Vision, 10(6), 5. doi:10.1167/10.6.5

Godijn, R., \& Theeuwes, J. (2002). Programming of endogenous and exogenous saccades: Evidence for a competitive integration model. Journal of Experimental Psychology: Human Perception and Performance, 28, 1039-1054. doi:10.1037/0096-1523.28.5.1039

Ishihara, S. (1993). Ishihara's tests for colour-blindness. Tokyo, Japan: Kanehara \& Co.

Lamy, D., Leber, A., \& Egeth, H. E. (2004). Effects of task relevance and stimulus-driven salience in feature-search mode. Journal of Experimental Psychology: Human Perception and Performance, 30, 1019-1031. doi:10.1037/0096-1523.30.6.1019

Leblanc, É., Prime, D. J., \& Jolicœur, P. (2008). Tracking the location of visuospatial attention in a contingent capture paradigm. Journal of Cognitive Neuroscience, 20, 657-671.

Miles, J. D., \& Proctor, R. W. (2011). Colour correspondence effects between controlled objects and targets. Quarterly Journal of Experimental Psychology, 64, 2044-2064. doi:10.1080/17470218. 2011.582130

Song, J.-H., \& Nakayama, K. (2006). Role of focal attention on latencies and trajectories of visually guided manual pointing. Journal of Vision, 6(9), 11. doi:10.1167/6.9.11

Song, J.-H., \& Nakayama, K. (2009). Hidden cognitive states revealed in choice reaching tasks. Trends in Cognitive Sciences, 13, 360-366. doi:10.1016/j.tics.2009.04.009

Spivey, M. J., Grosjean, M., \& Knoblich, G. (2005). Continuous attraction toward phonological competitors. Proceedings of the National Academy of Sciences, 102, 10393-10398.

Theeuwes, J., \& Godijn, R. (2002). Irrelevant singletons capture attention: Evidence from inhibition of return. Perception \& Psychophysics, 64, 764-770. doi:10.3758/BF03194743

Tipper, S. P., Howard, L. A., \& Houghton, G. (1998). Action-based mechanisms of attention. Philosophical Transactions of the Royal Society B, 353, 1385-1393.

Tipper, S. P., Lortie, C., \& Baylis, G. C. (1992). Selective reaching: Evidence for action-centered attention. Journal of Experimental Psychology: Human Perception and Performance, 18, 891-905. doi:10.1037/0096-1523.18.4.891

Welsh, T. N. (2011). The relationship between attentional capture and deviations in movement trajectories in a selective reaching task. Acta Psychologica, 137, 300-308. doi:10.1016/j.actpsy.2011.03.011

Welsh, T. N., Elliott, D., \& Weeks, D. J. (1999). Hand deviations towards distractors: Evidence for response competition. Experimental Brain Research, 127, 207-212.

Welsh, T. [N.], \& Elliott, D. (2004). Movement trajectories in the presence of a distracting stimulus: Evidence for a response activation model of selective reaching. Quarterly Journal of Experimental Psychology, 57A, 1031-1057. doi:10.1080/02724980343000666 